\title{
Working memory capacity and retrieval from long-term memory: the role of controlled search
}

\author{
Nash Unsworth • Gene A. Brewer • \\ Gregory J. Spillers
}

Published online: 3 October 2012

(C) Psychonomic Society, Inc. 2012

\begin{abstract}
In two experiments, the role of working memory capacity (WMC) in the controlled search of long-term memory was examined. Participants performed a prolonged category fluency task that required them to retrieve as many animals as possible in $5 \mathrm{~min}$. The results suggested that WMC differences arose in the numbers of animals retrieved, the numbers of clusters retrieved, and the rates of the retrieval (Exp. 1). However, no differences were found in terms of how participants initiated retrieval or in the nature of the clusters generated. Furthermore, an examination of differences in retrieval strategies suggested that high-WMC individuals were more strategic than low-WMC individuals and that these differences in retrieval strategies accounted for the overall differences in the numbers of animals retrieved. Additionally, presenting participants with retrieval cues eliminated WMC differences in the numbers of animals retrieved (Exp. 2). These results suggest that low-WMC individuals are less able than high-WMC individuals to select and utilize appropriate retrieval strategies to self-generate cues to access information in long-term memory. Collectively, the results are consistent with research suggesting that WMC is important for controlled search from long-term memory.
\end{abstract}

Keywords Working memory · Individual differences

Working memory, and individual differences in working memory capacity (WMC), has long been shown to be an important predictor of higher-order cognitive abilities such

N. Unsworth $(\bowtie) \cdot$ G. J. Spillers

Department of Psychology, University of Oregon,

Eugene, OR 97403, USA

e-mail: nashu@uoregon.edu

G. A. Brewer

Department of Psychology, Arizona State University,

Tempe, AZ, USA as intelligence (see Engle \& Kane, 2004). Previous work has suggested that retrieval of information from long-term memory is also an important process to working memory and is part of the reason for individual differences in WMC (Cowan et al., 2003; Healey \& Miyake, 2009; Kane \& Engle, 2000; D. L. Nelson \& Goodmon, 2003; Radvanksy \& Copeland, 2006; Rosen \& Engle, 1997; Unsworth \& Engle, 2007). In particular, recent correlational work has suggested that measures of working memory are moderately to strongly related to measures of long-term memory (Mogle, Lovett, Stawski, \& Sliwinski, 2008; Unsworth, 2010; Unsworth, Brewer, \& Spillers, 2009).

Despite initial evidence for a relation between WMC and long-term memory abilities, the reason for the relation is still not fully understood. Although it is likely that strategic encoding processes are important, we have previously suggested that controlled search is also an important reason for the relation between WMC and long-term memory (Spillers \& Unsworth, 2011; Unsworth, 2007). Our goal in the present study was to examine the relation between WMC and long-term memory by examining controlled search processes in a difficult and prolonged long-term memory task.

\section{Controlled search processes}

Voluntary recall from long-term memory (LTM) typically requires an active search of memory in which LTM is probed with various cues to attempt to access the desired information. This search process is thought to be dictated by both directed and random components (Shiffrin, 1970; Shiffrin \& Atkinson, 1969). The directed component refers to those strategic processes that are under direct control of the individual. These strategic control processes include setting up an overall retrieval plan, selecting various retrieval strategies, and selecting and generating appropriate cues 
to search memory with, as well as various monitoring strategies and decisions as to whether or not to continue searching (T. O. Nelson \& Narens, 1990). The random component refers to the probabilistic nature of the search process, in which a subset of information is activated by the cues (i.e., the search set), and representations are subsequently sampled (probabilistically with replacement) and recovered from this subset (Raaijmakers \& Shiffrin, 1980; Shiffrin, 1970). Thus, recall from LTM is dictated not only by various strategies that individuals bring to bear on the task, but also by the probabilistic nature of recall from LTM. The choice of which retrieval strategies to use is an important first step in the overall search process. As was noted by Koriat, Goldsmith, and Halamish (2008), "The strategy used to search memory determines in part the context of retrieval, the generation of additional retrieval cues, and the ways those cues are used to retrieve information from memory" (p. 311). Selecting and controlling appropriate retrieval strategies can influence the overall search process, as well as the ability to actually retrieve the target information.

One important search strategy is what is known as mediated retrieval (Koriat et al., 2008). In this strategy, one first attempts to generate general contextual information associated with the target information, and then attempts to use that information to generate more specific information that will lead to the target information. This notion of first using a general cue to generate information and then using the products of the search to further specify the search process is an integral component of various search models (Burgess \& Shallice, 1996; M. A. Conway \& Pleydell-Pearce, 2000; Norman \& Bobrow, 1979; Reiser, Black, \& Abelson, 1985; Whitten \& Leonard, 1981; Williams \& Hollan, 1981).

\section{Working memory capacity and controlled search}

Theoretically, controlled search processes are reliant on intact frontally mediated control processes such as the central executive in working memory or the supervisory attentional system (Baddeley \& Wilson, 1986; Burgess \& Shallice, 1996; M. A. Conway, 1992). It has been suggested that control is needed to select appropriate retrieval strategies, to generate appropriate contexts to search, to elaborate on the cues needed for search, to verify the products of the search, and to adequately use the products of the search to better focus the retrieval specification (M. A. Conway, 1992; Koriat et al., 2008; Raaijmakers \& Shiffrin, 1980; Shiffrin, 1970). Thus, working memory control processes should be of vital importance when one is attempting to strategically search LTM, and these working memory control processes should be especially important during retrieval strategy selection and cue-elaboration phases in which one must self-generate different contexts to search.
Recent work has suggested that working memory capacity (WMC) is needed for retrieval from LTM and that individual differences in WMC are partially due to differences in controlled search processes (Unsworth \& Engle, 2007). From the work above on controlled search processes, we have suggested that the covariation between WMC and measures of recall from LTM occurs because individual differences in WMC largely reflect differences in the directed components of the search process (Rosen \& Engle, 1997; Unsworth \& Engle, 2007). We have suggested that lowWMC individuals are poorer at generating retrieval cues to focus their search sets than are high-WMC individuals. Rosen and Engle found that high- and low-WMC individuals differed in their abilities to retrieve animal names from semantic memory during an extended verbal fluency task. Specifically, high-WMC individuals recalled more animal names, recalled more clusters of animal names, had larger cluster sizes, and recalled at a faster rate than did low-WMC individuals. Rosen and Engle suggested that high-WMC individuals were better at self-generating cues to access animal names than are low-WMC individuals, leading to better overall performance. Recently, in a large-scale individual-differences study, we extended the results of Rosen and Engle and found that a latent variable of WMC strongly predicted the total number of words generated in a number of verbal fluency tasks, as well as the number of clusters and the size of the clusters (Unsworth, Spillers, \& Brewer, 2011).

Although the results above provided initial evidence for the notion of WMC differences in controlled search processes, much more work is needed to better clarify and understand controlled search processes in general, as well as to examine what aspects of controlled search processes are reliant on intact WMC. Thus, there is a need to better examine how individuals strategically search their LTM systems. For example, if it is assumed that a key component of controlled search is the ability to select and appropriately utilize various search strategies, it seems likely that individuals who differ in WMC will also differ in their abilities to select and implement these strategies. That is, do high- and low-WMC individuals select different search strategies when asked to recall information from LTM? If so, does this impact how individuals search their LTM systems? Some recent work by Schelble, Therriault, and Miller (2012) has suggested that high- and low-WMC individuals differ in the use of retrieval strategies in fluency tasks and that these differences partially accounted for differences in recall levels, but more work is needed to examine these differences and whether differences in strategies also account for differences in other aspects of recall (such as the number of clusters generated). Likewise, if the generation of cues used to conduct the search is important and generation is facilitated in high-WMC individuals, then these individuals ought to retrieve items in clusters to a greater degree than do low-WMC 
individuals. Furthermore, if the main difference between highand low-WMC individuals in retrieval is due to the fact that low-WMC individuals are not as efficient as high-WMC individuals at spontaneously self-generating retrieval cues, then providing low-WMC individuals with retrieval cues should lead to performance similar to that of high-WMC individuals. In contrast, if high- and low-WMC individuals differ qualitatively in how they search LTM, simply by generating different retrieval cues, then providing retrieval cues should not change performance much.

Overall, in the present study we were interested in examining a number of important issues related to the role of WMC in controlled search from LTM. We examined whether WMC differences exist in the ability to self-generate retrieval cues and in the various search strategies used to generate cues. We were also interested in examining whether there are WMC differences in how search is carried out. Specifically, we were interested in examining whether high- and low-WMC individuals differ in how they begin the search process (i.e., what types of cues are used initially) and whether there are differences in the natures of the clusters generated by high- and lowWMC individuals in terms of the different situational or environmental contexts generated. To examine these issues, we had high- and low-WMC individuals perform a prolonged verbal fluency task in which they were required to generate exemplars from the category of animals for $5 \mathrm{~min}$. We chose this task because prior work has shown that individual differences in WMC are strongly related to overall performance in this task (e.g., Rosen \& Engle, 1997; Unsworth et al., 2011), and also because this type of task has been used previously to examine search processes in more detail (e.g., Graesser \& Mandler, 1978; Gruenewald \& Lockhead, 1980; Herrmann \& Pearle, 1981; Shiffrin, 1970; Walker \& Kintsch, 1985; Williams \& Hollan, 1981; Wixted \& Rohrer, 1994). Furthermore, prior work has shown that this task is related to other fluency tasks (Unsworth et al., 2011) and that overall WMC differences in this task are similar to those in other fluency tasks, such as autobiographical fluency (Unsworth, Spillers, \& Brewer, 2012). By using this type of task, we hoped to gain a better understanding of the nature of controlled search processes as well as of the nature of individual differences in WMC and controlled search.

\section{Experiment 1}

The purpose of Experiment 1 was to examine the role of WMC in controlled search processes. In particular, we investigated whether WMC differences arise in the ability to self-generate retrieval cues and in the strategies used to generate different retrieval cues. High- and low-WMC individuals were asked to generate exemplars from the category of animals for $5 \mathrm{~min}$. After attempting to generate animal names, participants provided information on each of their responses. This information was then used to determine whether participants clustered related information together (e.g., recalled zoo animals together), the number of these clusters, the size of these clusters, as well the interresponse times associated with recalling items. Furthermore, this information was used to determine the different types of contexts (e.g., pets, farm animals, or zoo animals) that participants might use in recalling animals, as well as the relative proportions with which different contexts get searched. Finally, following the coding of each response, participants filled out a questionnaire regarding the various search strategies that they used to perform the fluency task (see also Schelble et al., 2012). Note that although prior work had investigated the relation between WMC and performance on this type of task, the prior work had not specifically examined the nature of the clusters generated, nor potential WMC differences in search strategies. For example, in Rosen and Engle (1997), participants were told to generate animal names, and clusters were defined on the basis of an algorithm that looked for interresponse time differences between words. Likewise, Unsworth et al. (2011) examined clusters on the basis of the scoring methods of Troyer, Moscovitch, and Winocur (1997), in which independent raters rather than the participants themselves actually coded the data. Thus, in both cases there was no examination of the contents of the actual clusters to see whether high and low WMC differed qualitatively in the natures of the clusters that they generated.

\section{Method}

\section{Participants and WMC screening}

Participants were recruited from the participant pool at the University of Georgia. Individuals were selected on the basis of a composite of the three complex span tasks described below. Only participants falling in the highest (highWMC) and lowest (low-WMC) quartiles of the composite distribution were selected.

Operation span Participants solved a series of math operations while trying to remember a set of unrelated letters that were presented for $1 \mathrm{~s}$ each. Immediately after a letter was presented, the next operation was presented. Three trials of each list length (3-7) were presented, with the order of list lengths varying randomly. At recall, letters from the current set were recalled in the correct order by clicking on the appropriate letters. For all of the span measures, the score was the proportion of correct items in the correct position (see Unsworth, Heitz, Schrock, \& Engle, 2005, for more task details). 
Symmetry span Participants were required to recall sequences of red squares within a matrix while performing a symmetry judgment task. In the symmetry judgment task, participants were shown an $8 \times 8$ matrix with some squares filled in black. Participants decided whether the design was symmetrical about its vertical axis. The pattern was symmetrical half of the time. Immediately after determining whether the pattern was symmetrical, participants were presented with a $4 \times 4$ matrix with one of the cells filled in red for $650 \mathrm{~ms}$. At recall, participants recalled the sequence of red-square locations in the preceding displays, in the order that they had appeared, by clicking on the cells of an empty matrix. There were three trials of each list length, with list length ranging from 2 to 5 .

Reading span Participants were required to read sentences while trying to remember the same set of unrelated letters as for operation span. For this task, the participants read a sentence and determined whether or not the sentence made sense. Half of the sentences made sense, and the other half did not. Nonsense sentences were made by simply changing one word from an otherwise normal sentence. After participants had indicated whether the sentence made sense or not, they were presented with a letter for $1 \mathrm{~s}$. At recall, the letters from the current set were recalled in the correct order by clicking on the appropriate letters. There were three trials of each list length, with list length ranging from 3 to 7 .

Composite score For the composite score, scores for the three complex span tasks were $z$-transformed for each participant. These $z$ scores were then averaged together, and quartiles were computed from the averaged distribution. The participants were 21 high-WMC individuals $(z$-WMC $=$ $0.92, S D=0.20)$ and 21 low-WMC individuals $(z-\mathrm{WMC}=$ $-1.09, S D=0.43$ ), as determined by the composite measure. The mean age for both groups was 18.4 years.

\section{Animal fluency}

Participants were instructed that they should retrieve as many exemplars from the category of animals as possible in $5 \mathrm{~min}$. The participants were informed that they could retrieve the names of animals in any order that they wished; they were required to type in each animal name, and then press Enter to record the response. We instructed the participants that they needed to keep trying to retrieve animal names throughout the entire 5-min retrieval period. Following the retrieval task, the participants were given a spreadsheet containing their responses on the retrieval task, along with various categories for classification. These categories included pet, farm, forest, jungle/safari, aquatic, sport/team, mythical, and other. We instructed the participants to classify each response in terms of whether it belonged in one of the categories and to classify each animal according to all of the categories in which it fit. The participants were also instructed to indicate whether the items in succession were recalled together, and to indicate additional categories if needed. In this way, the participants indicated their own clusters (see also Buschke, 1977). In addition to the basic classification scheme, we also gave participants a column to provide a more detailed explanation for each animal name and encouraged them to provide as much information as possible. Thus, immediately after the retrieval task participants provided detailed information on their responses, and this information was used to code the data (Burgess \& Shallice, 1996). The participants were given as much time as they needed to perform the classification aspect of the experiment. All of the information obtained from this procedure was then used to determine how participants had organized their retrieval, in terms of different clusters based on shared contexts between successively retrieved items.

\section{Search strategy questionnaire}

Following the coding of their data, the participants also completed a brief questionnaire regarding any search strategies that they had used during the animal fluency task. First, they were asked in an open-ended format whether they had used any strategies to perform the animal fluency task, and if so, to describe the strategies in detail. Next, participants answered closed-ended questions regarding their strategies. They indicated (in order) whether they had used a visualization strategy, a semantic strategy, a rhyme strategy, a literature strategy, a strategy based on overall knowledge of animals, or no strategy, so that the results were based on random responding. The participants could indicate that they had used more than one strategy during the course of retrieval.

\section{Results}

The overall results, in the form of the cumulative number of animal names retrieved as a function of time and WMC, are shown in Fig. 1. An examination of these cumulative recall functions suggests a number of findings. First, high-WMC individuals retrieved more animal names than did lowWMC individuals. Second, high-WMC individuals seem to have retrieved names at a faster rate than did low-WMC individuals; that is, high-WMC individuals retrieved more items in the same amount of time as low-WMC individuals. Third, the rates of retrieval slowed toward the end of the retrieval period, despite the fact that individuals were still retrieving items. These results are consistent with those of Rosen and Engle (1997). 
Fig. 1 Mean cumulative numbers of items retrieved as a function of recall time (in seconds) and working memory capacity (WMC)

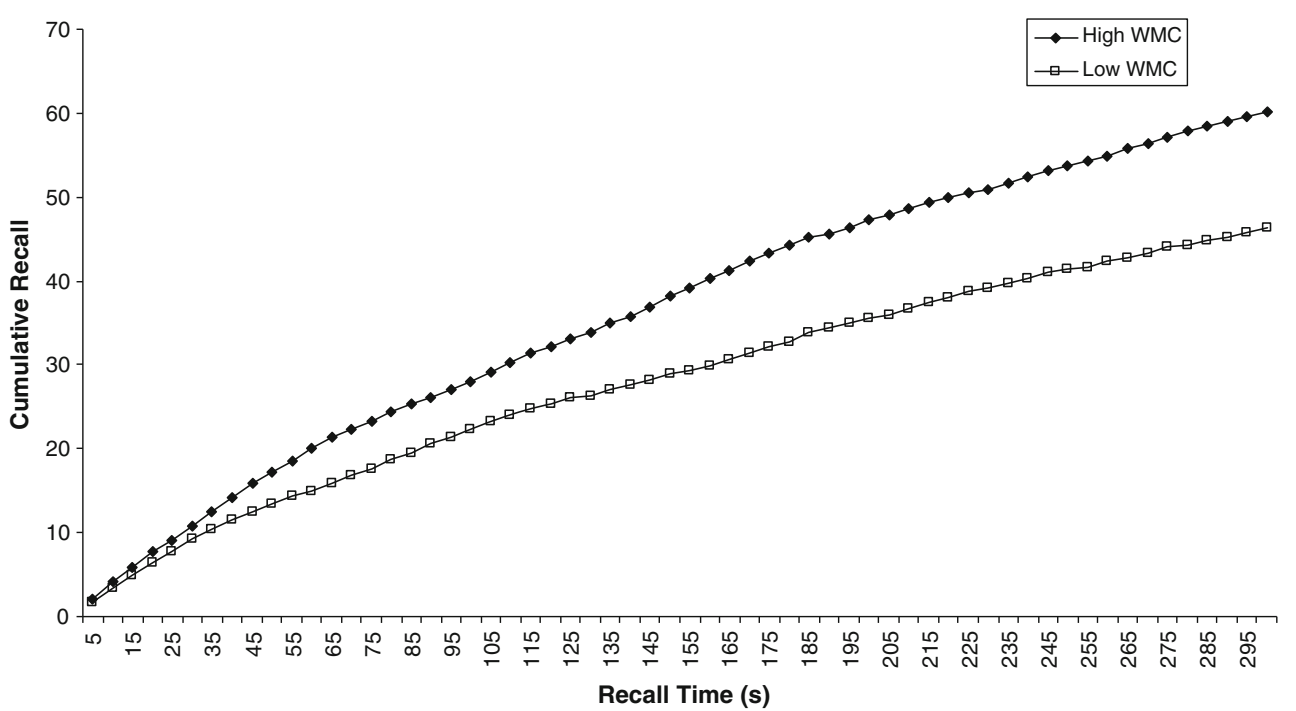

In order to examine these overall retrieval differences in more detail, we examined the total numbers of animals retrieved, the number of clusters that each individual retrieved, and the size of each cluster. Here, clusters refer to two or more items retrieved from the same context (e.g., pets) in succession, as indicated by the participants. Thus, the participants' own coding was used to determine the number and sizes of clusters. Importantly, this should give an indication of the number of contexts that were self-generated. As can be seen in Table 1, high-WMC individuals retrieved more animals, $t(40)=3.21, p<.01, \eta^{2}=.20$, and retrieved more clusters, $t(40)=2.48, p<.05, \eta^{2}=.13$, than did low-WMC individuals. There were no differences in the sizes of the clusters generated, $t(40)=0.66, p>.51, \eta^{2}=.01$. These results suggest that high-WMC individuals' retrieval was more organized than low-WMC individuals' retrieval. Indeed, an examination of the proportion of random items retrieved (i.e., items not associated with a cluster) suggested that low-WMC individuals retrieved a higher proportion of random items $(M=$ $.39, S E=.07)$ than did high-WMC individuals $(M=.20, S E=$ $.05), t(40)=2.17, p<.05, \eta^{2}=.11$. In fact, for many of these items, low-WMC individuals were more likely than high-WMC individuals to specifically indicate that the name "randomly popped" into their head. Note that although the number of animals retrieved and the number of clusters generated will be correlated, these measures are not redundant, given that it is possible for two individuals to retrieve different numbers of animals but to generate the same number of clusters. This is because individuals can also differ in the sizes of the clusters generated, as well as in the number of random items (i.e., items not associated with a cluster) retrieved.

An analysis of recall latency variables should provide additional evidence for differences in the efficiency of searching memory. In particular, an examination of first-recall latencies and interresponse times (IRTs) should provide important information regarding the ease of searching within contexts and accessing names. These measures were times to first keypress. An examination of recall latencies as a function of WMC suggested that high- and low-WMC individuals started out recalling at the same time, but high-WMC individuals recalled at a faster rate than did low-WMC individuals thereafter. ${ }^{1}$ Specifically, as is shown in Table 1 , high- and lowWMC individuals both started their recall after approximately $2.5 \mathrm{~s}$ (i.e., time to first recall), $t(40)=1.29, p>.20, \eta^{2}=.04$, but thereafter high-WMC individuals' IRTs were faster than low-WMC individuals', $t(40)=3.51, p<.01, \eta^{2}=.24$. These results are consistent with the notion that high-WMC individuals are more efficient at searching their memory than are lowWMC individuals, in that the cues that high-WMC individuals use focus their search better, leading to quicker access to the desired information.

Given the overall WMC differences, the next issue to examine was the nature of the retrieved items, in terms of what categories that they were from and whether high- and low-WMC individuals differed in the categories from which they retrieved the items. We first examined whether highand low-WMC individuals differed in how they initiated retrieval in terms of which categories they began with. To do this, we examined the probability of first recall as a function of the seven primary categories that the participants had used to code their data. Specifically, for the first item that each participant retrieved, we examined whether that item was coded by the participant as a pet, a farm animal, a forest animal, a jungle animal, an aquatic animal, an animal from a sports team, or a mythical animal. This should give an indication of how participants initiated their search. Shown in Fig. 2 are the resulting probability-of-first-recall (PFR) functions as a function of WMC and category. As can

\footnotetext{
${ }^{1}$ Prior to performing the fluency task, participants had completed a brief typing exercise (typing the words one to ten) to estimate their typing efficiency. There were no differences between high- and lowWMC individuals in typing speed in either experiment (both $t \mathrm{~s}<1$ ).
} 
Table 1 Retrieval measures for the entire 5-min retrieval period as a function of working memory capacity (WMC)

\begin{tabular}{|c|c|c|c|c|c|}
\hline \multirow[t]{2}{*}{ WMC } & \multicolumn{5}{|l|}{ Measure } \\
\hline & No. Retrieved & No. Clusters & Cluster Size & IRT & Time-to-First \\
\hline High & $61.26(3.19)$ & $13.52(0.87)$ & $4.07(0.22)$ & $4.99(0.23)$ & $2.43(0.22)$ \\
\hline Low & $47.29(2.98)$ & $10.71(0.72)$ & $3.85(0.26)$ & $6.75(0.44)$ & $2.82(0.20)$ \\
\hline
\end{tabular}

IRT, interresponse time. The IRT and time-to-first variables are measured in seconds. Standard errors are shown in parentheses

be seen, the vast majority of participants initiated retrieval with an animal considered to be a pet, with a much smaller proportion of participants initiating retrieval with a jungle animal. No participants initiated retrieval with a farm, aquatic, or mythical animal. In terms of individual differences in WMC, the results suggest that high- and low-WMC individuals initiated retrieval in similar manners, with most high- and low-WMC individuals initiating retrieval with a pet. These observations were supported by a $2(\mathrm{WMC}) \times 7$ (category) ANOVA. Specifically, we found a main effect of category, $F(6,240)=39.41, M S E=$ $.08, p<.01, \eta_{\mathrm{p}}{ }^{2}=.50$, but no effect of WMC, $p>.32$, and no interaction, $p>90$. Thus, there were no differences in how high- and low-WMC individuals initiated retrieval.

Next, we examined the proportion of retrieved items from each category as a function of WMC. Recall that prior studies had found differences in the number of category clusters retrieved as a function of WMC, but these studies had not examined the nature of these clusters and the nature of the retrieved items to see whether qualitative differences exist between high- and low-WMC individuals in terms of how they search. It is possible that WMC differences in retrieval in this type of task are due to differences in how participants search, with low-WMC individuals searching through a smaller set of categories (e.g., only pets), whereas high-WMC individuals might utilize a more sophisticated search strategy that results in a large search set composed of multiple categories. In order to examine this question, for each individual we computed the proportion of animals retrieved that were classified as belonging to one of the categories. As is shown in Table 2, the majority of items retrieved seemed to come from the jungle and aquatic categories, followed by pets, forest animals, and farm animals, with many fewer items coming from the sport or mythical categories. Additionally, as is shown in Table 2, high- and low-WMC individuals seemed to retrieve similar proportions of items from each category. These observations were supported by a 2 (WMC) $\times 7$ (category) ANOVA. There was a main effect of category, $F(6,240)=28.74, M S E=.01$, $p<.01, \eta_{\mathrm{p}}{ }^{2}=.42$, but no effect of WMC, $p>.30$, and no interaction, $p>.79$. Thus, although high-WMC individuals retrieved more items and more clusters of items than did low-WMC individuals, the groups did not differ in the natures of the items retrieved.

For our final set of analyses, we examined the retrieval strategy reports in hopes of better understanding how highand low-WMC individuals differed in their search abilities. First, we examined the open-ended question of strategy use. Two raters examined the responses and determined that seven general strategies were reported. These included a visual location strategy, in which participants suggested that they visualized different locations (e.g., a zoo) and then imagined different animals associated with that location; a
Fig. 2 Probabilities of first recall (PFR) as a function of category and working memory capacity (WMC). Error bars reflect one standard error of the mean

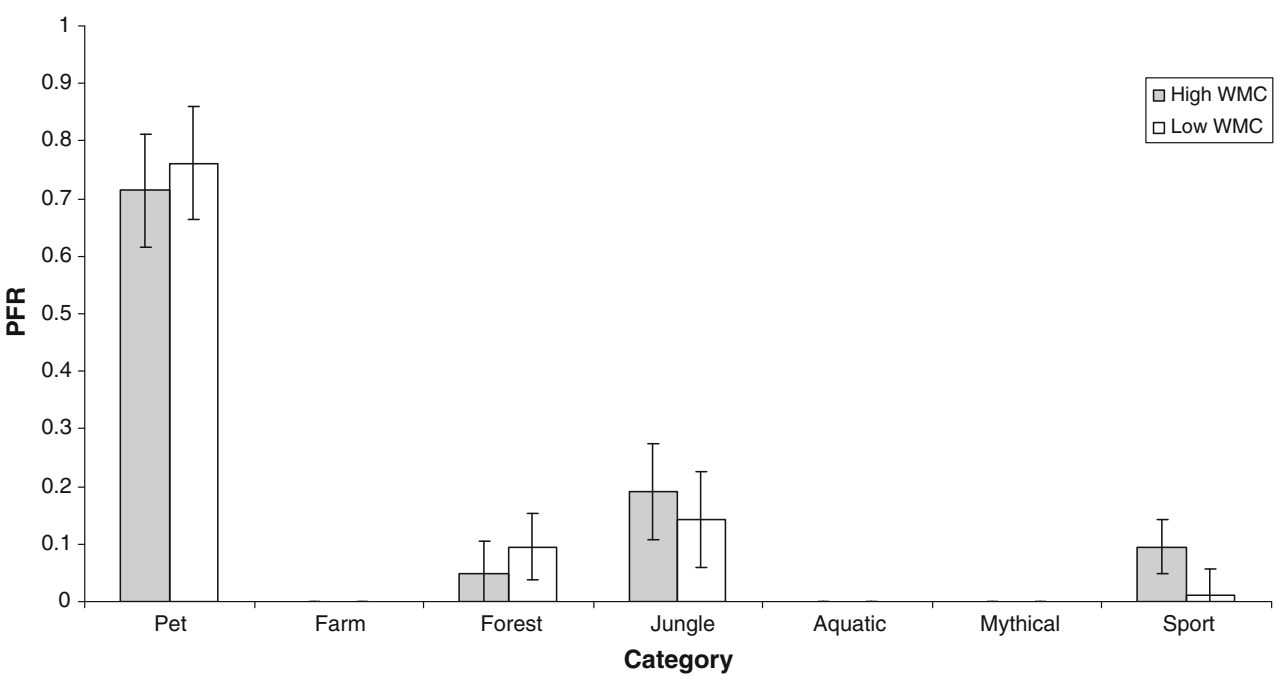


Table 2 Proportions of items retrieved from each category as a function of working memory capacity (WMC)

\begin{tabular}{|c|c|c|c|c|c|c|c|}
\hline \multirow[t]{2}{*}{ WMC } & \multicolumn{7}{|l|}{ Category } \\
\hline & Pet & Farm & Forest & Jungle & Aquatic & Mythical & Sport \\
\hline High & $0.17(0.02)$ & $0.12(0.02)$ & $0.17(0.02)$ & $0.23(0.02)$ & $0.25(0.02)$ & $0.01(0.01)$ & $0.05(0.02)$ \\
\hline Low & $0.19(0.03)$ & $0.11(0.02)$ & $0.15(0.03)$ & $0.25(0.04)$ & $0.24(0.04)$ & $0.01(0.01)$ & $0.04(0.02)$ \\
\hline
\end{tabular}

Standard errors are shown in parentheses

general-to-specific strategy, in which participants suggested that they first thought of an overall general type of animal (e.g., birds) and then listed all of the animals associated with that general type (e.g., robin, canary, eagle); an alphabetic strategy, in which participants named animals in alphabetical order; an opposite strategy, in which participants generated an animal and then tried to think of the opposite of that animal; a link-to-previous strategy, in which participants intentionally used the just-recalled item as a cue to recall additional items; a recent strategy, in which participants tried to think of animals that they had encountered recently; and finally, no strategy, in which participants specifically noted that the words just randomly popped into their heads. For each participant, responses were coded in terms of these strategies. Interrater agreement was high ( $>95 \%)$, and disagreements were resolved.

As can be seen in Table 3, the strategies most likely to be used across participants were the visual and link-to-previous strategies, followed by the general-to-specific strategy. Very few participants reported using the alphabetic, opposite, or recent strategies. However, quite a few participants reported at times using no strategy, and simply recalling items randomly. In terms of WMC, the main differences seemed to be that high-WMC individuals were more likely to use a general-tospecific strategy than were low-WMC individuals, and that low-WMC individuals were more likely to use the random/no strategy than were high-WMC individuals. These observations were supported by a $2(\mathrm{WMC}) \times 7$ (strategy) ANOVA. We found a main effect of strategy, $F(6,240)=11.13, M S E=$ $.16, p<.01, \eta_{\mathrm{p}}{ }^{2}=.22$. Follow-up analyses suggested that there was no difference in the proportions of reported use for the visual and link-to-previous strategies $(p>.20)$, and that the visual strategy was reported more frequently than any of the other strategies (all $p \mathrm{~s}<.05$ ). Furthermore, there were no differences in the use of the general-to-specific strategy, the link-to-previous strategy, and the random/no strategy (all $p$ s $<$ .16), but all of these strategies were reported more frequently than the alphabetic, opposite, and recent strategies (all $p$ s $<$ $.05)$. The main effect of WMC was not significant, $F<1$, but the WMC $\times$ Strategy interaction was significant, $F(6,240)=$ $2.54, M S E=.16, p<.05, \eta_{\mathrm{p}}{ }^{2}=.06$. High-WMC individuals reported using the general-to-specific strategy more than did low-WMC individuals, $t(40)=2.45, p<.05, \eta^{2}=.13$, and low-WMC individuals reported using a random/no strategy more frequently than did high-WMC individuals, $t(40)=2.28$, $p<.05, \eta^{2}=.11$. No other WMC-related differences emerged in the proportions of strategy use, all $p \mathrm{~s}>.16$. Thus, although high- and low-WMC individuals reported using very similar strategies, there were clear differences in two of the strategies that high- and low-WMC individuals reported, suggesting that high-WMC individuals were more strategic in their search, at times, than low-WMC individuals.

Next, we examined the closed-ended questions that participants answered regarding the strategies that they used. Specifically, participants indicated whether they had used a visualization strategy, a semantic strategy, a rhyme strategy, a literature strategy, a strategy based on overall knowledge of animals, or no strategy, so that the results were based on random responding. Participants could indicate that they used more than one strategy during the course of retrieval. Shown in Table 4 are the proportions of reported use for each strategy as a function of WMC. As can be seen, the most frequently reported strategies were semantic, visual, and random/no strategy, with intermediate numbers of participants reporting using a knowledge strategy or literature strategy, and even fewer participants reporting using a rhyme strategy. In terms of WMC, differences arose in the use of the knowledge

Table 3 Proportions of reported strategy use for open-ended questions as a function of strategy and working memory capacity (WMC)

\begin{tabular}{|c|c|c|c|c|c|c|c|}
\hline \multirow[t]{2}{*}{ WMC } & \multicolumn{7}{|l|}{ Strategy } \\
\hline & Visual & GenSpec & Alpha & Opposite & Link & Recent & Random \\
\hline High & $0.57(0.11)$ & $0.48(0.11)$ & $0.05(0.05)$ & $0.00(0.00)$ & $0.48(0.11)$ & $0.00(0.00)$ & $0.10(0.07)$ \\
\hline Low & $0.52(0.11)$ & $0.14(0.08)$ & $0.05(0.05)$ & $0.05(0.05)$ & $0.33(0.11)$ & $0.10(0.07)$ & $0.38(0.11)$ \\
\hline
\end{tabular}

GenSpec, general-to-specific strategy; Alpha, alphabetic strategy; Link, link-to-previous strategy; Random, words recalled randomly/no strategy. The proportions of strategies sum to greater than 1.0 because the participants could report using more than one strategy. Standard errors are shown in parentheses 
Table 4 Proportions of reported strategy use for closed-ended questions as a function of strategy and working memory capacity (WMC)

\begin{tabular}{|c|c|c|c|c|c|c|}
\hline \multirow[t]{2}{*}{ WMC } & \multicolumn{6}{|l|}{ Strategy } \\
\hline & Visual & Semantic & Rhyme & Literature & Knowledge & Random \\
\hline High & $0.67(0.11)$ & $0.95(0.05)$ & $0.00(0.00)$ & $0.29(0.10)$ & $0.29(0.10)$ & $0.43(0.11)$ \\
\hline Low & $0.71(0.10)$ & $0.81(0.09)$ & $0.10(0.07)$ & $0.24(0.10)$ & $0.05(0.05)$ & $0.76(0.10)$ \\
\hline
\end{tabular}

The proportions of strategies sum to greater than 1.0 because the participants could report using more than one strategy. Standard errors are shown in parentheses

strategy and the random/no strategy. These observations were supported by a 2 (WMC) $\times 6$ (strategy) ANOVA, which revealed a main effect of strategy, $F(5,200)=27.88, M S E=$ $.16, p<.01, \eta_{\mathrm{p}}{ }^{2}=.41$. The main effect of WMC was not significant, $F<1$, but the WMC $\times$ Strategy interaction was significant, $F(5,200)=2.59, M S E=.16, p<.05, \eta_{\mathrm{p}}{ }^{2}=.06$. High-WMC individuals reported using the knowledge strategy more than low-WMC individuals did, $t(40)=2.13, p<.05$, $\eta^{2}=.10$, and low-WMC individuals reported using a random/ no strategy more frequently than high-WMC individuals did, $t$ $(40)=2.28, p<.05, \eta^{2}=.12$. No other WMC-related differences emerged in the proportions of strategy use, all $p \mathrm{~s}>.16$. The results from the closed-ended questions were very similar to those from the open-ended questions, suggesting that highand low-WMC individuals used several similar strategies, but that high-WMC individuals were more likely to use their knowledge base while searching than were low-WMC individuals, and low-WMC individuals were more likely to rely on random responding than were high-WMC individuals.

\section{Discussion}

The results from Experiment 1 demonstrated a number of findings. Replicating prior work, we found that in a fixed amount of time, high-WMC individuals retrieved more items, more clusters of items, and at a faster rate than did low-WMC individuals (Rosen \& Engle, 1997). The present study also demonstrated a number of novel findings. For example, it was found that highWMC individuals retrieved roughly $80 \%$ of their items in clusters, whereas low-WMC individuals retrieved roughly $60 \%$ of their items in clusters. Examining how participant initiated retrieval suggested that high- and low-WMC individuals initiated retrieval in similar fashions, by retrieving predominantly pets. Furthermore, examining the nature of the items retrieved suggested that high- and low-WMC individuals tended to retrieve in similar fashions, in that high- and lowWMC individuals retrieved similar proportions of items from each of the different categories. Finally, although high- and lowWMC individuals reported using very similar strategies overall, high-WMC individuals tended to rely more on their knowledge base to engage in general-to-specific searches than did low-
WMC individuals, and low-WMC individuals were more likely than high-WMC individuals to engage in a random search in which items were passively retrieved (see also Schelble et al., 2012). Collectively, these results suggest that high-WMC individuals are more likely to use specific search strategies (as opposed to no strategy) than are low-WMC individuals, which helps in the self-generation of cues to search, resulting in more recalled items overall and more clusters of related items. Consistent with this notion, we found that the WMC difference in the total numbers of items retrieved was eliminated when differences in strategies were covaried out. Specifically, when the general-to-specific, knowledge-base, and random strategies were entered in as covariates, the effect of WMC on the total number of items retrieved was no longer significant, $F(1,36)=$ $1.80, M S E=155.22, p>.18, \eta_{\mathrm{p}}{ }^{2}=.04$. Likewise, the effect of WMC on the number of clusters retrieved was no longer significant when strategies were taken into account, $F<1, \eta_{\mathrm{p}}{ }^{2}=$ .01. Thus, high- and low-WMC individuals seemed to differ in their abilities to use strategies to self-generate cues during search, but high- and low-WMC individuals did not differ in the nature of the search, with both initiating search in the same way and both demonstrating similar search profiles.

\section{Experiment 2}

Experiment 2 was conducted to better examine the notion that high- and low-WMC individuals differ in the ability to selfgenerate cues during search of LTM. In particular, although the results of Experiment 1 are suggestive of the possibility that high- and low-WMC individuals differ in the fundamental ability to self-generate cues, more direct evidence was needed. That is, if the main difference between high- and low-WMC individuals in recall is due to the fact that low-WMC individuals are not as efficient as high-WMC individuals at spontaneously self-generating retrieval cues, then providing low-WMC individuals with retrieval cues should lead to performance similar to that of high-WMC individuals. In contrast, if highand low-WMC individuals differ qualitatively in how they search LTM, by simply generating different retrieval cues, then providing retrieval cues should not change performance much. To better examine this possibility, we brought in new high- and 
low-WMC individuals to perform the same basic animal fluency task as in Experiment 1. In one group, new high- and lowWMC individuals performed the exact same animal fluency task as in Experiment 1. This served as the control condition. Another group of high- and low-WMC individuals performed the same animal fluency task as in Experiment 1, but the participants were presented with a set of retrieval cues that remained onscreen at all times. Specifically, participants were presented with subcategories of animals (pets, farm animals, forest animals, etc.) at the top of the recall screen. These participants were informed that subcategory cues of animals would be provided onscreen to help them recall animals if needed. Thus, the participants were provided with cues, but they did not have to use them if they did not want to. This will be referred to as the free-cue condition. Finally, a third group of high- and low-WMC individuals performed the same fluency task as did the other groups, but now each WMC group was given a different category cue (the same cues as the free-cue condition) onscreen for 20 s (e.g., Randolph, Braun, Goldberg, \& Chase, 1993). The participants were instructed to recall animals from that subcategory only during these $20 \mathrm{~s}$. After $20 \mathrm{~s}$, a new cue appeared. This will be referred to as the forced-cue condition. Providing participants with cues should not only boost overall recall levels, but also eliminate WMC differences if the main difference between high- and low-WMC individuals is the ability to self-generate retrieval cues.

\section{Method}

Participants and WMC screening

The participants were recruited from the participant pool at the University of Georgia. Individuals were selected on the basis of a $z$-score composite of the same three complex span tasks as in Experiment 1. Only participants falling in the highest (highWMC individuals) and lowest (low-WMC individuals) quartiles of the composite distribution were selected.

Composite score For the composite score, scores for the three complex span tasks were $z$-transformed for each participant. These $z$ scores were then averaged together, and quartiles were computed from the averaged distribution. The participants were 53 high-WMC individuals $(z-\mathrm{WMC}=0.89, S D=0.54)$ and 53 low-WMC individuals $(z-\mathrm{WMC}=-1.28, S D=0.73)$, as determined by the composite measure. Of these, 19 highand 19 low-WMC individuals were assigned to the control condition, 18 high- and 18 low-WMC individuals were in the free-cue condition, and 16 high- and 16 low-WMC individuals were in the forced-cue condition. The participants were randomly assigned to the conditions.
Animal fluency

The participants were instructed that they would be recalling as many exemplars from the category of animals as possible in $5 \mathrm{~min}$. They were required to type in each animal name and then press Enter to record the response, and they were instructed that they needed to keep trying to recall animal names throughout the entire 5-min recall period. The control condition was exactly the same as the task in Experiment 1. The free-cue condition was exactly the same as the control condition, except that a set of subcategory retrieval cues was presented onscreen at all times, and participants were informed that they could use those cues to aid recall if they needed. The subcategory cues were pets, farm animals, jungle animals, sea creatures, zoo animals, forest animals, sports teams, types of birds, types of fish, reptiles, canines, felines, rodents, Australian animals, and mythical creatures. These cues were chosen on the basis of the results from Experiment 1, of similar cues from Randolph et al. (1993), and of pilot work. In the forced-cue condition, participants were told that they would be recalling as many exemplars from the category of animals as possible in $5 \mathrm{~min}$, but that every $20 \mathrm{~s}$ a new subcategory cue would be presented, and they were instructed to only recall animals that matched that cue and to continue recalling such animals until a new cue was presented. Like the control fluency task, the forced-cued task lasted for $5 \mathrm{~min}$. Thus, in all three conditions participants were required to recall animals, but the extent to which cues were presented and the extent to which participants were required to use the cues varied. Strategy data were not recorded in this experiment, given that some of the participants were given explicit cues to use during recall.

\section{Results}

The results are shown in Fig. 3. As can be seen, performance increased from the control (no-cue) condition to the forcedcue condition. Additionally, in terms of WMC differences, the control condition replicated Experiment 1, suggesting large WMC differences, with high-WMC individuals retrieving more animals than did low-WMC individuals. However, these differences were reduced in the free-cue condition, and were ultimately eliminated in the forced-cue condition. These observations were supported by a 2 $($ WMC) $\times 3$ (condition) ANOVA that revealed a main effect of condition, $F(2,100)=50.86, M S E=91.35, p<.01, \eta_{\mathrm{p}}{ }^{2}=$ .50 , suggesting that performance was higher in the forcedcue condition $(M=75.56, S E=1.69)$ than in the free-cue condition $(M=60.89, S E=1.59)$, which in turn was higher than performance in the control condition $(M=52.58, S E=$ 1.56 ), all $p \mathrm{~s}<.01$. We also found a main effect of WMC, $F$ $(1,100)=8.21, M S E=91.35, p<.01, \eta_{\mathrm{p}}^{2}=.08$, with highWMC individuals $(M=65.68, S E=1.32)$ retrieving more 
Fig. 3 Mean numbers of items recalled as a function of condition and working memory capacity (WMC). Error bars reflect one standard error of the mean

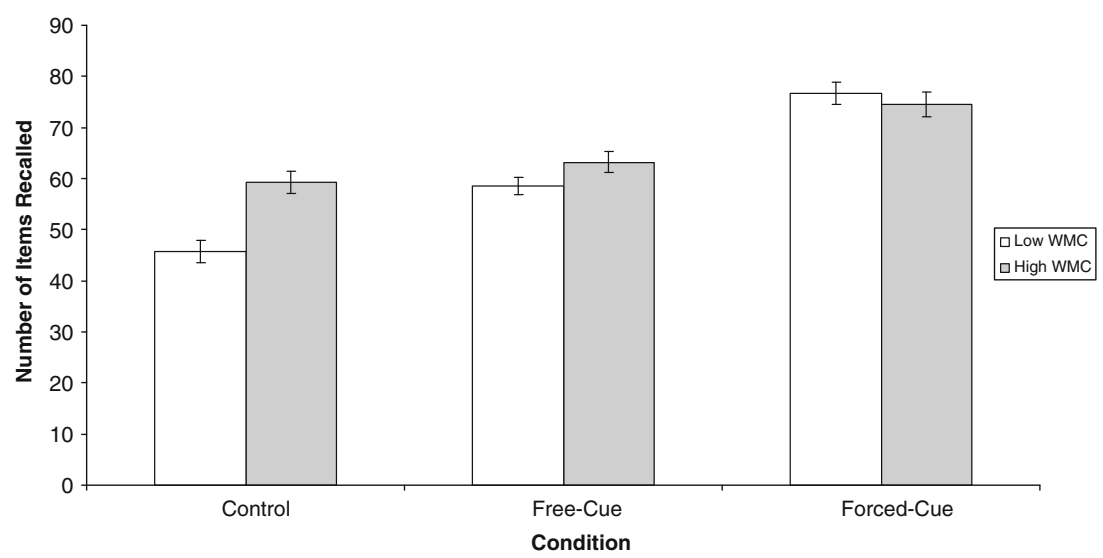

animals than did low-WMC individuals $(M=60.34, S E=$ 1.32). Importantly, these two factors interacted, $F(2,100)=$ $6.05, M S E=91.35, p<.01, \eta_{\mathrm{p}}{ }^{2}=.11$, suggesting that WMC differences decreased (and were eliminated) as participants were required to use cues. Specifically, in the control condition, high-WMC individuals retrieved more animals than did low-WMC individuals, replicating Experiment 1, $t(36)=$ $4.81, p<.01, \eta^{2}=.39$. This difference between high- and low-WMC individuals was reduced, but still significant, in the free-cue condition, $t(34)=2.50, p<.05, \eta^{2}=.16$. Finally, WMC differences were eliminated in the forcedcue condition, $t(30)=0.48, p<.63, \eta^{2}=.01$. Accordingly, low-WMC individuals benefited more from cues than did high-WMC individuals, increasing their retrieval by roughly 31 items, whereas high-WMC individuals only increased their retrieval by roughly 15 items. Thus, providing participants with cues (and ensuring that they used those cues) eliminated WMC differences in retrieval.

\section{Discussion}

The results from Experiment 2 were relatively straightforward. Overall, presenting participants with retrieval cues in a verbal fluency task increased performance (Randolph et al., 1993). In terms of WMC, when no cues were provided in a prolonged verbal fluency task, high-WMC individuals retrieved substantially more items than did low-WMC individuals. When cues were presented and participants were free to use those cues, WMC differences were reduced but not eliminated. However, if cues were presented and participants were required to use those cues, WMC differences were eliminated. Thus, a main difference between high- and low-WMC individuals in terms of retrieval from LTM seems to be the ability to self-generate cues to guide the search process. When no cues are provided, low-WMC individuals are less able to use retrieval strategies to generate cues to aid in the search than are high-WMC individuals. However, when retrieval cues are presented (and participants are required to use them) and participants are no longer required to self-generate their own cues, WMC differences are eliminated. Note that nothing was particularly special about the cues used in the present study. Rather, what was important was that we created a situation in which participants did not have to self-generate their own cues to retrieve the desired information. One aspect of the present design that seems important in this regard is the fact that not only were participants given specific cues, but also the amount of time to retrieve animals from those cues was not excessively long. When given only $20 \mathrm{~s}$ to recall as many pets from LTM as possible, participants tended to retrieve high-frequency pets relatively automatically. Had we given participants much longer to retrieve animals (such as 5 min per cue), one would expect that WMC differences would remain. This is because after high-frequency exemplars are retrieved relatively automatically, participants would need to engage in a more strategic search of LTM for specific categories of pets over a prolonged amount of time. Thus, it was not simply the act of providing participants with cues that was important, but the fact that these cues allowed participants to retrieve items relatively automatically, without needing to selfgenerate their own cues. Future work will be needed to examine these issues better across a number of prolonged LTM tasks.

\section{General discussion}

In two experiments, we examined the role of WMC in retrieval from LTM. Specifically, high- and low-WMC individuals performed a prolonged verbal fluency task in which exemplars from the category of animals had to be retrieved over $5 \mathrm{~min}$. In Experiment 1, we found that high-WMC individuals retrieved more animals and more clusters of animals, and retrieved at a faster rate, than did low-WMC individuals. Despite these differences in overall levels of retrieval, high- and lowWMC individuals did not differ qualitatively in how they searched LTM. Specifically, high- and low-WMC individuals initiated retrieval in the same manner and retrieved the same proportion of animals from similar categories. Furthermore, although high- and low-WMC individuals tended to use 
similar retrieval strategies, high-WMC individuals reported using a general-specific strategy based on their overall knowledge base more than low-WMC individuals did, and lowWMC individuals reported using random responding (i.e., no strategy) more often than high-WMC individuals did. Importantly, these differences in reported retrieval strategy use accounted for the relations between WMC and both the number of animals and the number of clusters retrieved. These results suggested that high-WMC individuals are better able than low-WMC individuals to select and use retrieval strategies (see also Schelble et al., 2012) to self-generate cues to use during search, but that high- and low-WMC individuals do not differ in the natures of their search processes.

The notion that high-WMC individuals are better at selfgenerating retrieval cues was directly examined in Experiment 2 , in which high- and low-WMC individuals performed the fluency task in the presence or absence of retrieval cues. When no cues were present, high-WMC individuals outperformed low-WMC individuals, consistent with Experiment 1. However, when retrieval cues were present and participants were required to use them, performance was boosted, and high- and low-WMC individuals retrieved the same number of animals. Thus, these results suggest that WMC differences in retrieval from LTM are due to differences in retrieval failures, whereby low-WMC individuals are less able to select and use retrieval strategies to self-generate retrieval cues.

The present results add to a growing body of research suggesting that WMC is important for controlled search processes. The results suggest that WMC is related to the ability to select and use particular retrieval strategies and to the ability to self-generate retrieval cues leading to clusters of related items. At the same time, the results suggest that WMC is not necessarily related to how participants search LTM, given that WMC was not related to how participants initiated search, nor is it related to the different contexts that participants recall from. Thus, WMC is not needed in all aspects of search. These results can be interpreted within prior models of controlled search, which have suggested that participants first select a retrieval strategy and then use that strategy to self-generate appropriate cues to aid in the search of LTM (mediated retrieval; Burgess \& Shallice, 1996; M. A. Conway \& PleydellPearce, 2000; Koriat et al., 2008; Norman \& Bobrow, 1979; Reiser et al., 1985; Whitten \& Leonard, 1981; Williams \& Hollan, 1981). Specifically, we suggest that high- and lowWMC individuals likely start out recalling high-frequency exemplars based on fairly spontaneous passive recall (Nickerson, 1981). However, given the prolonged nature of the task, random/passive recall will cease to produce many results, and participants will likely switch to a more strategic/ active approach. In this case, both high- and low-WMC individuals will select various retrieval strategies throughout the task. The most common of these in the present task were visual and semantic strategies. Importantly, on some occasions, high-WMC individuals will be more likely than low-WMC individuals to rely on their knowledge base and to utilize a general-to-specific strategy, whereas low-WMC individuals will be more likely to revert back to random/passive recall than will high-WMC individuals. In these cases, highWMC individuals will continue to generate clusters of items, whereas low-WMC individuals will likely recall single items, leading to superior recall for high-WMC individuals. Thus, the ability to select and utilize appropriate strategies to generate retrieval cues throughout the recall period, rather than reverting back to random recall, seems to differentiate highand low-WMC individuals. When retrieval cues are provided and it is ensured that participants utilize those cues, the need to select retrieval strategies and generate cues is eliminated and high and low WMC individuals demonstrate equivalent levels of recall. An interesting prediction from this theory is that providing participants with specific retrieval cues that are not particularly suited to the task would simultaneously eliminate WMC differences and reduce recall levels. For example, if high- and low-WMC individuals are forced to use alphabetic retrieval cues, which are known to lead to lower levels of recall than are freely generated cues (e.g., Gronlund \& Shiffrin, 1986), we would expect that WMC differences would be eliminated, with both high- and low-WMC individuals demonstrating lower levels of recall than in the control condition. This suggests that retrieval cues do not have to be optimal to get rid of WMC differences, but rather that the need to use strategic processes must be eliminated before high- and lowWMC individuals are equated.

A potential alternative explanation for the present results is that perhaps high- and low-WMC individuals differ in mental processing speed, whereby low-WMC individuals are slower overall than high-WMC individuals. This explanation suggests that if given enough time, low-WMC individuals should reach levels of retrieval similar to those of high-WMC individuals. Evidence consistent with this notion is that in Experiment 1, high-WMC individuals had faster IRTs than did lowWMC individuals. Thus, it is possible that differences in processing speed partially accounted for the present results. Although we did not specifically measure processing speed in the present study, we do not think that differences in speed accounted for the present results. Prior work examining cumulative recall functions in both animal fluency tasks and episodic free-recall tasks has demonstrated that high- and low-WMC individuals do not reach the same levels of asymptotic recall (Rosen \& Engle, 1997; Unsworth, 2007). In particular, in Rosen and Engle's study, high- and low-WMC individuals were given $15 \mathrm{~min}$ to retrieve animals, and even after $15 \mathrm{~min}$, low-WMC individuals were not close to reaching the same levels of retrieval as high-WMC individuals. In fact, differences in the numbers of animals retrieved increased throughout the $15 \mathrm{~min}$. Additionally, as was noted in note 1, our high- and low-WMC individuals did not differ in overall 
typing speed. Furthermore, although many studies have found a relation between WMC and processing speed in aging studies, the relation between WMC and processing speed in younger adults is much weaker (see, e.g., A. R. A. Conway, Cowan, Bunting, Therriault, \& Minkoff, 2002; Hedden, Lautenschlager, \& Park, 2005; Redick, Unsworth, Kelly, \& Engle, 2012; Unsworth et al., 2011). In particular, in a largescale latent variable study, we found that although measures of processing speed and WMC both predicted performance on multiple measures of verbal fluency, the processing speed and WMC latent factors were not correlated (Unsworth et al., 2011). Thus, it seems unlikely that differences in processing speed could account for the present results, although future work will be needed to better test these hypotheses.

An additional alternative explanation is that perhaps highand low-WMC individuals differ in motivation, such that low-WMC individuals are simply not motivated to perform well, leading to lower scores on both the WMC measures and the fluency task. Although we did not directly measure motivation in the present study, we also do not think that differences in motivation or effort accounted for the present results. Much prior work has examined the notion that individual differences in WMC are simply due to differences in effort or motivation, and this prior work has suggested that this explanation is unlikely. Specifically, Heitz, Schrock, Payne, and Engle (2008) examined high- and low-WMC individuals on a version of the reading span task in which the participants were paid on the basis of their performance. Heitz et al. found that providing participants with a monetary incentive increased overall performance and increased pupil size (thought to be a physiological index of effort), and these increases were equivalent for high- and low-WMC individuals. Thus, increasing motivation and effort increased performance equally for high- and low-WMC individuals, and low-WMC individuals did not reach the same levels of performance as did high-WMC individuals. Furthermore, if high- and low-WMC individuals had overall baseline differences in motivation, one would expect WMC differences on nearly all tasks. However, this is not the case, as many WMC studies have demonstrated conditions in which high- and low-WMC individuals have equivalent performance, and other conditions in which they differ (see Unsworth \& Engle, 2007). Importantly, the conditions in which no differences are found are not simply easier. For example, Kane and Engle (2003) demonstrated similar levels of interference on the Stroop task with all incongruent trials. Likewise, Kane, Poole, Tuholski, and Engle (2006) demonstrated equivalent performance between high- and low-WMC individuals in a number of quite difficult visual search tasks. Thus, it seems unlikely that differences in motivation could account for the present results. Although motivation and effort clearly do influence performance, these variables typically do not interact with WMC.
Collectively, the present results suggest that high-WMC individuals are better at strategically searching their memory systems than are low-WMC individuals, because high-WMC individuals are better at dynamically utilizing search strategies, self-generating cues that specify the correct target items (Unsworth, 2007), and elaborating on the cues needed for search (Spillers \& Unsworth, 2011; Unsworth \& Engle, 2007). Of course, these results are preliminary, and more work will be needed to directly examine the role of WMC in the strategic search of LTM and to examine a number of important questions. In particular, while most of the prior work has specifically focused on aspects of recall such as clustering and temporal dynamics, far less work has directly examined search strategies. An important endeavor for future research will be to examine better how individuals utilize search strategies to access information from their LTM and whether there are individual differences in these abilities. Although individuals clearly differ in WMC and retrieval from LTM, future work will be needed to better understand these differences.

Author Note We thank Philip Beaman, Ken Malmberg, and an anonymous reviewer for many valuable comments on a previous version of the manuscript.

\section{References}

Baddeley, A. D., \& Wilson, B. (1986). Amnesia, autobiographical memory, and confabulation. In D. C. Rubin (Ed.), Autobiographical memory (pp. 225-252). Cambridge: Cambridge University Press.

Burgess, P. W., \& Shallice, T. (1996). Confabulation and the control of recollection. Memory, 4, 359-411.

Buschke, H. (1977). Two-dimensional recall: Immediate identification of clusters in episodic and semantic memory. Journal of Verbal Learning and Verbal Behavior, 16, 201-215.

Conway, A. R. A., Cowan, N., Bunting, M. F., Therriault, D. J., \& Minkoff, S. R. B. (2002). A latent variable analysis of working memory capacity, short-term memory capacity, processing speed, and general fluid intelligence. Intelligence, 30, 163-183.

Conway, M. A. (1992). A structural model of autobiographical memory. In M. A. Conway, D. C. Rubin, H. Spinnler, \& E. W. Wagenaar (Eds.), Theoretical perspectives on autobiographical memory (pp. 167-194). Dordrecht: Kluwer.

Conway, M. A., \& Pleydell-Pearce, C. W. (2000). The construction of autobiographical memories in the self-memory system. Psychological Review, 107, 261-288. doi:10.1037/0033-295X.107.2.261

Cowan, N., Towse, J. N., Hamilton, Z., Saults, J. S., Elliot, E. M., Lacey, J. F., \& Hitch, G. J. (2003). Children's working-memory processes: A response-timing analysis. Journal of Experimental Psychology: General, 132, 113-132.

Engle, R. W., \& Kane, M. J. (2004). Executive attention, working memory capacity, and a two- factor theory of cognitive control. In B. H. Ross (Ed.), The psychology of learning and motivation, Vol. 44 (pp. 145-199). San Diego: Academic Press.

Graesser, A. C., \& Mandler, G. (1978). Limited processing capacity constrains the storage of unrelated sets of words and retrieval from natural categories. Journal of Experimental Psychology: Human Learning and Memory, 4, 86-100. 
Gronlund, S. D., \& Shiffrin, R. M. (1986). Retrieval strategies in recall of natural categories and categorized lists. Journal of Experimental Psychology: Learning, Memory, and Cognition, 12, 550-561.

Gruenewald, P. J., \& Lockhead, G. R. (1980). The free recall of category examples. Journal of Experimental Psychology: Human Learning and Memory, 6, 225-240.

Healey, M. K., \& Miyake, M. (2009). The role of attention during retrieval in working memory span: A dual-task study. Quarterly Journal of Experimental Psychology, 62, 733-745.

Hedden, T., Lautenschlager, G., \& Park, D. C. (2005). Contributions of processing ability and knowledge to verbal memory tasks across the adult lifespan. Quarterly Journal of Experimental Psychology, 58, 169-190.

Heitz, R. P., Schrock, J. C., Payne, T. W., \& Engle, R. W. (2008). Effects of incentive on working memory capacity: Behavioral and pupillometric data. Psychophysiology, 45, 119-129.

Herrmann, D. J., \& Pearle, P. M. (1981). The proper role of clusters in mathematical models of continuous recall. Journal of Mathematical Psychology, 24, 139-162.

Kane, M. J., \& Engle, R. W. (2000). Working memory capacity, proactive interference, and divided attention: Limits on longterm retrieval. Journal of Experimental Psychology: Learning, Memory, and Cognition, 26, 333-358.

Kane, M. J., \& Engle, R. W. (2003). Working-memory capacity and the control of attention: The contributions of goal neglect, response competition, and task set to Stroop interference. Journal of Experimental Psychology. General, 132, 47-70. doi:10.1037/00963445.132.1.47

Kane, M. J., Poole, B. J., Tuholski, S. W., \& Engle, R. W. (2006). Working memory capacity and the top-down control of visual search: Exploring the boundaries of "executive attention. Journal of Experimental Psychology: Learning, Memory, and Cognition, 32, 749-777.

Koriat, A., Goldsmith, M., \& Halamish, V. (2008). Controlled processes in voluntary remembering. In J. H. Byrne \& H. L. Roediger III (Eds.), Learning and memory: A comprehensive reference: Vol. 2. Cognitive psychology of memory (pp. 307-324). Amsterdam: Elsevier.

Mogle, J. A., Lovett, B. J., Stawski, R. S., \& Sliwinski, M. J. (2008). What's so special about working memory? An examination of the relationship among working memory, secondary memory, and fluid intelligence. Psychological Science, 19, 1071-1077.

Nelson, D. L., \& Goodmon, L. B. (2003). Disrupting attention: The need for retrieval cues in working memory theories. Memory and Cognition, 31, 65-76. doi:10.3758/BF03196083

Nelson, T. O., \& Narens, L. (1990). Metamemory: A theoretical framework and new findings. In G. H. Bower (Ed.), The psychology of learning and motivation: Advances in research and theory (Vol. 24, pp. 125-173). San Diego: Academic Press.

Nickerson, R. S. (1981). Motivated retrieval from archival memory. In G. H. Bower (Ed.), Nebraska Symposium of Motivation (Vol. 28, pp. 73-119). Lincoln: University of Nebraska Press.

Norman, D. A., \& Bobrow, D. G. (1979). Descriptions: An intermediate stage in memory retrieval. Cognitive Psychology, 11, 107-123. doi:10.1016/0010-0285(79)90006-9

Raaijmakers, J. G. W., \& Shiffrin, R. M. (1980). SAM: A theory of probabilistic search of associative memory. In G. H. Bower (Ed.), The psychology of learning and motivation Vol. 14 (pp. 207-262). New York: Academic Press. doi:10.1016/S0079-7421(08)60162-0

Radvansky, G. A., \& Copeland, D. E. (2006). Memory retrieval and interference: Working memory issues. Journal of Memory and Language, 55, 33-46.

Randolph, C., Braun, A. R., Goldberg, T. E., \& Chase, T. N. (1993). Semantic fluency in Alzheimer's, Parkinson's, and Huntington's disease: Dissociation of storage and retrieval failures. Neuropsychology, 7, 82-88.

Redick, T. S., Unsworth, N., Kelly, A. J., \& Engle, R. W. (2012). Faster, smarter? Working memory capacity and perceptual speed in relation to fluid intelligence. Journal of Cognitive Psychology. doi:10.1080/20445911.2012.704359

Reiser, B. J., Black, J. B., \& Abelson, R. P. (1985). Knowledge structures in the organization and retrieval of autobiographical memories. Cognitive Psychology, 17, 89-137.

Rosen, V. M., \& Engle, R. W. (1997). The role of working memory capacity in retrieval. Journal of Experimental Psychology. General, 126, 211-227.

Schelble, J. L., Therriault, D. J., \& Miller, M. D. (2012). Classifying retrieval strategies as a function of working memory. Memory and Cognition, 40, 218-230. doi:10.3758/s13421-011-0149-1

Shiffrin, R. M. (1970). Memory search. In D. A. Norman (Ed.), Models of human memory (pp. 375-447). New York: Academic Press.

Shiffrin, R. M., \& Atkinson, R. C. (1969). Storage and retrieval processes in long-term memory. Psychological Review, 76, 179193. doi: $10.1037 / \mathrm{h} 0027277$

Spillers, G. J., \& Unsworth, N. (2011). Variation in working memory capacity and temporal-contextual retrieval from episodic memory. Journal of Experimental Psychology: Learning, Memory, and Cognition, 37, 1532-1539. doi:10.1037/a0024852

Troyer, A. K., Moscovitch, M., \& Winocur, G. (1997). Clustering and switching as two components of verbal fluency: Evidence from younger and older healthy adults. Neuropsychology, 11, 138-146.

Unsworth, N. (2007). Individual differences in working memory capacity and episodic retrieval: Examining the dynamics of delayed and continuous distractor free recall. Journal of Experimental Psychology: Learning, Memory, and Cognition, 33, 1020-1034.

Unsworth, N. (2010). On the division of working memory and longterm memory and their relation to intelligence: A latent variable analysis. Acta Psychologica, 134, 16-28. doi:10.1016/ j.actpsy.2009.11.010

Unsworth, N., Brewer, G. A., \& Spillers, G. J. (2009). There's more to the working memory-fluid intelligence relationship than just secondary memory. Psychonomic Bulletin and Review, 16, 931-937. doi:10.3758/PBR.16.5.931

Unsworth, N., \& Engle, R. W. (2007). The nature of individual differences in working memory capacity: Active maintenance in primary memory and controlled search from secondary memory. Psychological Review, 114, 104-132. doi:10.1037/0033295X.114.1.104

Unsworth, N., Heitz, R. P., Schrock, J. C., \& Engle, R. W. (2005). An automated version of the operation span task. Behavior Research Methods, 37, 498-505. doi:10.3758/BF03192720

Unsworth, N., Spillers, G. J., \& Brewer, G. A. (2011). Variation in verbal fluency: A latent variable analysis of switching, clustering, and overall performance. Quarterly Journal of Experimental Psychology, 64, 447-466.

Unsworth, N., Spillers, G. J., \& Brewer, G. A. (2012). The role of working memory capacity in autobiographical retrieval: Individual differences in strategic search. Memory, 20, 167-176.

Walker, W. H., \& Kintsch, W. (1985). Automatic and strategic aspects of knowledge retrieval. Cognitive Science, 9, 261-283.

Whitten, W. B., \& Leonard, J. M. (1981). Directed search through autobiographical memory. Memory and Cognition, 9, 566-579.

Williams, M. D., \& Hollan, J. D. (1981). The process of retrieval from very long-term memory. Cognitive Science, 5, 87-119.

Wixted, J. T., \& Rohrer, D. (1994). Analyzing the dynamics of free recall: An intergrative review of the empirical literature. Psychonomic Bulletin and Review, 1, 89-106. doi:10.3758/BF03200763 